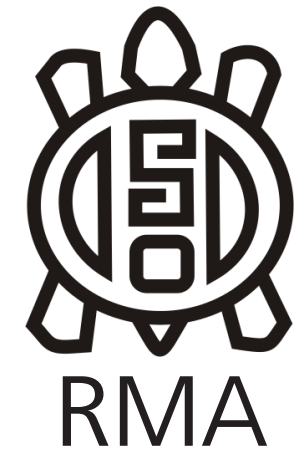

Dossier

\title{
El uso del cuarzo en la Sierra El Alto-Ancasti (Catamarca, Argentina). Experimentación y casos arqueológicos
}

The use of quartz in the El Alto-Ancasti mountains (Catamarca, Argentina). Experimentation and archaeological cases

\author{
Débora Egea* e Ignacio Gerola** \\ *Centro de Investigaciones y Transferencia de Catamarca (CITCA), CONICET. Catamarca, \\ Argentina. E-mail: deb.egea@gmail.com \\ **Instituto de Arqueología, Facultad de Filosofía y Letras, Universidad de Buenos Aires, \\ Argentina. E-mail:ignaciogerola@gmail.com
}

\begin{abstract}
Resumen
Los estudios de la tecnología lítica de las ocupaciones de la segunda mitad del primer milenio de la era en la Sierra de El Alto-Ancasti nos han permitido pensar en una producción caracterizada por una manufactura simple, de bajo grado de formatización de los instrumentos, donde destaca el aprovechamiento del cuarzo como materia prima. El cuarzo presenta características intrínsecas propias de su composición que dificultan la identificación de rasgos antrópicos diagnósticos que nos otorguen información sobre las historias de producción y las diferentes estrategias implementadas por los talladores a la hora de confeccionar su instrumental. Debido a esto se emprendió un abordaje experimental orientado a someter a prueba hipótesis explicativas sobre el comportamiento de esta materia prima ante diferentes técnicas de talla. Pretendemos probar la potencialidad de la experimentación para inferir comportamientos que no pueden ser observados directamente con los análisis descriptivos que venimos realizando en los conjuntos líticos de la zona.
\end{abstract}

Palabras Clave: Cuarzo; Arqueología experimental; Sierra de El Alto-Ancasti; Materias primas; Prácticas.

\begin{abstract}
The studies of the lithic technology of the occupations of the second half of the era's first millennium in El AltoAncasti mountains have allowed us to infer a production characterized by a simple manufacture, with low degree of formatization of the instruments, in which the use of quartz as raw material stands out.

However, quartz presents intrinsic characteristics of its composition which makes the identification of diagnostic anthropic features, that give us information about the production histories and the different strategies implemented by the carvers, difficult to diagnose. Due to this, an experimental approach oriented to the treatment of this raw material was undertaken, through different knapping techniques. We intend to test the potential of experimentation to infer behaviors that cannot be directly observed with the descriptive analyzes that we have been carrying out in the lithic assemblages of the zone.
\end{abstract}

Keywords: Quartz; Experimental archaeology; El Alto-Ancasti mountains; Raw material; Practices.

En los últimos años se ha producido un avance en el conocimiento de la tecnología lítica de la Sierra de El Alto-Ancasti mediante el estudio de diversos sitios arqueológicos de la zona. Estás investigaciones muestran que la producción lítica del área se ha caracterizado por una manufactura simple, de bajo grado de formatización de instrumentos, y por la selección predominante del cuarzo como materia prima, alcanzando representaciones del $98 \%$ en cada conjunto. La amplia y abundante distribución de esta roca en el terreno sería una de las principales explicaciones de su utilización (Egea, 2018; Gerola, 2018).

No obstante, el cuarzo presenta ciertos rasgos característicos, como su dureza y su estructura interna con fisuras, que dificultan tanto la talla controlada durante la producción cómo también la identificación de los rasgos antrópicos diagnósticos, esenciales para abordar las historias de producción y las diferentes estrategias de talla involucradas (Pautassi y Sario, 2014, entre otros). Debido a esto, en el último tiempo se han realizado y publicado numerosos estudios sobre el uso particular del cuarzo, tanto desde el punto de vista tecnológico como funcional (Brizuela, 2018; Knutsson, Knutsson, Taipale, Tallavaara y Darmark, 2015; Mansur y Lasa, 2005; entre otros).

Sin embargo, ante ciertas dudas que nos surgieron durante el proceso de análisis del material lítico de la zona, vemos la necesidad de re-interpretar el mismo a partir de nuevos datos, específicamente mediante la realización de un trabajo de experimentación orientado 
al tratamiento del cuarzo.

En particular, el objetivo es abordar los resultados obtenidos mediante la aplicación de diversas técnicas de talla, evaluando las características específicas de los productos de talla conseguidos en las primeras etapas de la producción lítica, tanto en la reducción del núcleo como la obtención de formas base. De esta forma, buscamos identificar rasgos diagnósticos propios de la talla de esta materia prima, explorar y precisar el potencial de cada técnica, poder ampliar la información que obtenemos de los conjuntos líticos y re-evaluar el material proveniente de los sitios arqueológicos estudiados.

El uso de la arqueología experimental nos permite generar información útil para verificar hipótesis sobre la formación del registro arqueológico. Así, a partir de experimentos de reproducción y replicación es posible inferir comportamientos que no pueden ser observados directamente en los restos arqueológicos. En relación a la dificultad que ofrece el cuarzo en la identificación de rasgos diagnósticos, el análisis experimental de la gestión de materias primas permite estudiar la influencia de las propiedades de cada recurso lítico en el desarrollo de los procesos técnicos y distinguir las características de los productos resultantes (Morgado y Baena, 2011; Nami, 2000).

\section{Ocupaciones analizadas}

La sierra de El Alto-Ancasti se ubica en la franja sudoriental de la provincia de Catamarca. En el área se pueden caracterizar dos paisajes diferentes y complementarios. El bosque serrano, que se desarrolla entre los 700/800 y 1400 msnm, y los pastizales de altura, ubicados entre los 1400 y los 1900 msnm.

Las poblaciones humanas del área habrían desarrollado las estrategias y tecnologías adecuadas para la explotación de los recursos locales y el desarrollo de producción agrícola y pastoril. En tanto, exhiben una inversión considerable en infraestructura agraria y habitacional (Gordillo, Zucarelli y Eguia, 2017; Quesada, Gastaldi y Granizo, 2012).

Hasta ahora hemos estudiado el material lítico de seis sitios arqueológicos, que se ubican a lo largo de la sierra. Las ocupaciones han sido fechadas en la segunda mitad del primer milenio de la era (500-1000 AD). El Taco 19 y Rodeo de los Indios son dos conjuntos habitacionales asociados a terrazas de cultivo que se encuentran emplazados en los pastizales de altura. En el ambiente de bosque serrano se localizan los asentamientos habitacionales Oyola 50, y Guayamba 2. También dentro del bosque serrano se encuentran Casa Pintada y Oyola 7 , dos cuevas con arte rupestre.

\section{La producción lítica en la sierra}

El cuarzo se presenta en las cercanías de los seis sitios analizados. En el bosque serrano se localiza tanto en afloramientos, o vetas, como también en forma de bochones redondeados desperdigados sobre la superficie del terreno inmediato a los sitios. En tanto, en los pastizales de altura las potenciales canteras de esta materia prima se ubican a 300 y $420 \mathrm{~m}$ en el caso de $\mathrm{El}$ Taco 19 y a 207 m de Rodeo de los indios.

La Tabla 1 compara la información proveniente del estudio de los productos de talla de los seis sitios analizados. El material fue abordado mediante un análisis tecnomorfológico y morfológico funcional macroscópico, siguiendo la propuesta de Aschero (1975, 1983). ${ }^{1}$

En todos los sitios los productos de talla muestran un porcentaje de fragmentación relativamente alto (entre 29\%-65\%). Sobresalen las lascas angulares (35\%-69\%) y hay porcentajes relevantes de desechos indiferenciados (8\%-22\%). Los tipos de talones predominantes corresponden a los talones lisos (43\%-81\%). Además, en casi todos los conjuntos hay porcentajes relevantes de talones filiformes (1\%-14\%) y puntiformes (3\%-16\%).

En general, hay una mayor representación de los desechos de tamaños pequeños (14\%-92\%) y módulos de longitud/anchura medianos (39\%-72\%), aunque se observa una alta variabilidad general. Los espesores también son diversos, predominando los espesores delgados (46\%-50\%) en todos los sitios a excepción de El Taco 19 (16\%).

Se pudo observar en los conjuntos algunos especímenes que presentan características que indican la utilización de talla bipolar, como ser la ausencia de talones, presencia de talones filiformes o puntiformes, lascas gajo de naranja, plataformas de percusión muy machacadas o con varios puntos de percusión y algún caso de estrías enfrentadas.

Los núcleos registrados en su mayoría son de lascados aislados. No se pudieron diferenciar los núcleos desbastados mediante percusión directa de los desbastados por talla bipolar. En El Taco 19, además, fueron identificados cinco percutores. En el caso de los sitios con arte rupestre no se recuperaron percutores ni núcleos (Egea, 2018; Gerola, 2018).

\section{Pruebas experimentales}

Se tallaron siete nódulos de cuarzo cuyos tamaños oscilaban los $50 \mathrm{~cm}$ de diámetro, con formas irregulares. Estos fueron seleccionados de cercanías del sitio Oyola 7 por su tamaño y transportabilidad. Tres de los nódulos

\footnotetext{
${ }^{1}$ Se incorporan solo los desechos de talla y formas base no retocadas ya que es lo que se puede comparar con la muestra experimental, debido a que no se avanzó con la formatización de instrumentos en la experimentación realizada.
} 
Tabla 1. Datos obtenidos de los conjuntos arqueológicos analizados.

Table 1. Data obtained from the archaeological set analyzed.

\begin{tabular}{|c|c|c|c|c|c|c|c|}
\hline \multirow{3}{*}{ Variables } & \multirow{3}{*}{$\begin{array}{c}\text { Estados de } \\
\text { variables }\end{array}$} & \multicolumn{6}{|c|}{ Sitios Arqueologicos } \\
\hline & & \multicolumn{2}{|c|}{ Habitacionales - pastizal } & \multicolumn{2}{|c|}{$\begin{array}{c}\text { Habitacionales- bosque } \\
\text { serrano }\end{array}$} & \multicolumn{2}{|c|}{$\begin{array}{c}\text { Cuevas - bosque } \\
\text { serrano }\end{array}$} \\
\hline & & $\begin{array}{l}\text { ET 19 } \\
(n=543)\end{array}$ & $\begin{array}{c}\text { R3 } \\
(n=110)\end{array}$ & OY50 $(n=1039)$ & $\mathrm{G} 2(\mathrm{n}=1058)$ & $\begin{array}{c}\mathrm{CP} \\
(\mathrm{n}=85)\end{array}$ & $\begin{array}{c}\text { OY7 } \\
(n=612)\end{array}$ \\
\hline \multirow{2}{*}{ Estado } & Entero & $36 \%$ & $43 \%$ & $49 \%$ & $63 \%$ & $70 \%$ & $35 \%$ \\
\hline & Fracturado & $64 \%$ & $57 \%$ & $51 \%$ & $37 \%$ & $30 \%$ & $65 \%$ \\
\hline \multirow{6}{*}{ Tipo } & Lasca angular & $69 \%$ & $35 \%$ & $44 \%$ & $41 \%$ & $67 \%$ & $37 \%$ \\
\hline & Lasca de arista & $1 \%$ & $2 \%$ & $23 \%$ & $6 \%$ & $4 \%$ & $18 \%$ \\
\hline & $\begin{array}{c}\text { Lasca no } \\
\text { diferenciada }\end{array}$ & $4 \%$ & $7 \%$ & $6 \%$ & $21 \%$ & $6 \%$ & $20 \%$ \\
\hline & $\begin{array}{l}\text { Lasca gajo de } \\
\text { naranja }\end{array}$ & $9 \%$ & $8 \%$ & $0 \%$ & $5 \%$ & $3 \%$ & $0 \%$ \\
\hline & Otros & $3 \%$ & $26 \%$ & $18 \%$ & $9 \%$ & $12 \%$ & $14 \%$ \\
\hline & Indiferenciados & $14 \%$ & $22 \%$ & $9 \%$ & $18 \%$ & $8 \%$ & $11 \%$ \\
\hline \multirow{4}{*}{$\begin{array}{l}\text { Tipo de } \\
\text { Talón }\end{array}$} & Filiforme & $9 \%$ & $1 \%$ & $14 \%$ & $7 \%$ & $13 \%$ & $8 \%$ \\
\hline & Puntiforme & $3 \%$ & $11 \%$ & $5 \%$ & $12 \%$ & $16 \%$ & $7 \%$ \\
\hline & Liso & $79 \%$ & $67 \%$ & $77 \%$ & $43 \%$ & $68 \%$ & $81 \%$ \\
\hline & Otros & $9 \%$ & $21 \%$ & $2 \%$ & $38 \%$ & $3 \%$ & $4 \%$ \\
\hline \multirow{3}{*}{ Tamaño } & Grandes & $10 \%$ & $0 \%$ & $4 \%$ & $1 \%$ & $0 \%$ & $1 \%$ \\
\hline & Medianos & $76 \%$ & $19 \%$ & $51 \%$ & $11 \%$ & $8 \%$ & $26 \%$ \\
\hline & Pequeño & $14 \%$ & $81 \%$ & $45 \%$ & $88 \%$ & $92 \%$ & $73 \%$ \\
\hline \multirow{3}{*}{$\begin{array}{l}\text { Modulo } \\
\text { longitud/ } \\
\text { anchura }\end{array}$} & Corto & $45 \%$ & $27 \%$ & $37 \%$ & $34 \%$ & $18 \%$ & $59 \%$ \\
\hline & Mediano & $51 \%$ & $67 \%$ & $57 \%$ & $56 \%$ & $71 \%$ & $39 \%$ \\
\hline & Laminar & $4 \%$ & $6 \%$ & $6 \%$ & $10 \%$ & $11 \%$ & $2 \%$ \\
\hline \multirow{4}{*}{ Espesor } & muy delgado & $2 \%$ & $6 \%$ & $14 \%$ & $35 \%$ & $50 \%$ & $21 \%$ \\
\hline & delgado & $17 \%$ & $49 \%$ & $49 \%$ & $46 \%$ & $46 \%$ & $50 \%$ \\
\hline & grueso & $60 \%$ & $44 \%$ & $31 \%$ & $17 \%$ & $4 \%$ & $25 \%$ \\
\hline & muy grueso & $21 \%$ & $1 \%$ & $6 \%$ & $2 \%$ & $0 \%$ & $4 \%$ \\
\hline
\end{tabular}

se trabajaron mediante talla directa y cuatro mediante talla bipolar, con el objetivo de regularizar plataformas de percusión y obtener formas base. Se utilizaron percutores de granito y cuarcita de $560 \mathrm{~g}$ en el caso de la talla directa, y de 820 y $930 \mathrm{~g}$ en el caso de la talla bipolar. Los conjuntos experimentales resultantes se analizaron a través de metodologías cuantitativas. Las variables tomadas en consideración fueron: estado y tipo de desecho, tipo del talón, y tamaño, módulo y espesor de cada una de las piezas (Tabla 2).

Con la aplicación de talla directa el total de productos obtenidos fue de 312 (208 lascas y 104 indiferenciados) mientras que con reducción bipolar se consiguieron 79 lascas. Los productos de la percusión directa presentaron un bajo porcentaje de fragmentación (22\%), mientras que los de talla bipolar mostraron un alto porcentaje de piezas fracturadas (78\%). También pudimos observar la gran cantidad de polvillo generada producto de la aplicación de ambas técnicas y la alta presencia de microlascas, muchas de ellas sin rasgos diagnósticos.
Los tipos de lascas fueron, con ambas técnicas de talla, mayormente angulares (directa 21\%/ bipolar 51\%). Con percusión directa, los productos de talla mostraron principalmente talones lisos (79\%), mientras que los de producción bipolar exhiben talones filiformes (81\%) y puntiformes (19\%) y algunas piezas con machacado en ambos extremos.

En cuanto a las variables dimensionales, los tamaños representados son mayores en la talla directa que en el caso de la talla bipolar. Los módulos son muy variados en la talla bipolar, en tanto que tienden a cortos (52\%) en la talla directa. Finalmente, los espesores son mayores en la talla directa que en la talla bipolar (Egea, 2018).

\section{Comparación de datos}

La comparación entre los resultados obtenidos mediante la experimentación y aquella información proveniente del análisis de los materiales arqueológicos, nos permite 
Tabla 2. Datos obtenidos del conjunto experimental analizado.

Table 2. Data obtained from the experimental set analyzed.

\begin{tabular}{|c|c|c|c|}
\hline \multirow{2}{*}{ Variables } & \multirow{2}{*}{$\begin{array}{c}\text { Estados de } \\
\text { variables }\end{array}$} & \multicolumn{2}{|c|}{ Experimentacion } \\
\hline & & $\begin{array}{c}\text { Talla directa } \\
(n=312)\end{array}$ & $\begin{array}{c}\text { Talla Bipolar } \\
(\mathrm{n}=79)\end{array}$ \\
\hline \multirow{2}{*}{ Estado } & Entero & $78 \%$ & $21 \%$ \\
\hline & Fracturado & $22 \%$ & $79 \%$ \\
\hline \multirow{6}{*}{ Tipo } & Lasca angular & $21 \%$ & $51 \%$ \\
\hline & Lasca de arista & $13 \%$ & $15 \%$ \\
\hline & $\begin{array}{l}\text { Lasca no } \\
\text { diferenciada }\end{array}$ & $4 \%$ & $0 \%$ \\
\hline & $\begin{array}{l}\text { Lasca gajo de } \\
\text { naranja }\end{array}$ & $0 \%$ & $14 \%$ \\
\hline & Otros & $25 \%$ & $21 \%$ \\
\hline & Indiferenciados & $33 \%$ & $0 \%$ \\
\hline \multirow{4}{*}{ Tipo de Talón } & Filiforme & $5 \%$ & $81 \%$ \\
\hline & Puntiforme & $14 \%$ & $19 \%$ \\
\hline & Liso & $78 \%$ & $0 \%$ \\
\hline & Otros & $3 \%$ & $0 \%$ \\
\hline \multirow{3}{*}{ Tamaño } & Grandes & $28 \%$ & $0 \%$ \\
\hline & Medianos & $60 \%$ & $38 \%$ \\
\hline & Pequeño & $12 \%$ & $62 \%$ \\
\hline \multirow{3}{*}{$\begin{array}{l}\text { Modulo } \\
\text { longitud/ } \\
\text { anchura }\end{array}$} & Corto & $52 \%$ & $35 \%$ \\
\hline & Mediano & $39 \%$ & $39 \%$ \\
\hline & Laminar & $2 \%$ & $25 \%$ \\
\hline \multirow{4}{*}{ Espesor } & muy delgado & $7 \%$ & $48 \%$ \\
\hline & delgado & $28 \%$ & $37 \%$ \\
\hline & grueso & $47 \%$ & $15 \%$ \\
\hline & muy grueso & $18 \%$ & $0 \%$ \\
\hline
\end{tabular}

inferir algunos aspectos particulares de la producción lítica en cuarzo de los sitios estudiados.

Resulta claro que en el proceso de producción de formas base existe un muy alto porcentaje de fragmentación y de desechos indiferenciados, así como gran cantidad de polvillo y fragmentos muy pequeños, sin rasgos diagnósticos. Lo que explica la abundante presencia de estas características en los conjuntos arqueológicos. Esta característica estaría vinculada a la dificultad para controlar la fractura del cuarzo, así como también a la presencia de irregularidades internas o procesos de oxidación de la roca (Pautassi y Sario, 2014; Prous, 2009). El porcentaje de fragmentación es más cercano a la talla bipolar en Oyola 7 (65\%), El Taco 19 (64\%) y Rodeo 3 (57\%), siendo menor y similar a los porcentajes experimentales de la talla directa en Guayamba 2 (70\%) y Casa Pintada (30\%).

En los experimentos de talla bipolar se observó que los desechos presentan atributos típicos de este tipo de talla, tales como marcas de contragolpe distales, talones filiformes o puntiformes. También se obtuvieron lascas gajo de naranja. Aspectos que habíamos notado en algunos de los conjuntos arqueológicos.
Lo que nos permite pensar para el caso de Oyola 7 o Casa Pintada, donde el porcentaje de talones filiformes es significativo, que esto podría deberse al uso de este tipo de percusión. Sin embargo, las lascas gajo de naranja, diagnosticas de la talla bipolar, se presentan en menores (o nulas) cantidades en las cuevas, y aumentan su representación en los asentamientos ubicados en los pastizales de altura.

Los talones lisos predominan en todos los asentamientos, lo que remarca la relevancia de la talla directa en los sitios, aunque se distingue la presencia de talones filiformes, vinculados a la talla bipolar, en todos los casos, destacando en Oyola 50 (14\%).

En los experimentos de talla bipolar no se detectaron, a diferencia de las piezas interpretadas como bipolares de los conjuntos arqueológicos, mayoría de módulos alargados o la ausencia de talones, lo que nos obliga a revisar ulteriores interpretaciones del material arqueológico basadas en estos aspectos.

Una de las principales observaciones sobre la técnica bipolar fue la excesiva fuerza necesaria para obtener lascas, además requiere varios golpes para poder lograr las mismas, lo que explica los extremos machacados en 
aquellos especímenes identificados en El Taco 19 y Oyola 50, por ejemplo. Además, logramos ver una vinculación entre menores espesores y la técnica bipolar, característica también registrada por otros investigadores en este tipo de percusión (De la Peña, 2015; Sanches Yustos, Diez Martín, Domínguez-Rodrigo y Tarriño Vinagre, 2012, entre otros).

Los espesores son mayores, por lo tanto, más similares a la talla directa, en los asentamientos de los pastizales de altura, El Taco 19 (58\%) y Rodeo 3 (44\%), mientras que predominan los muy delgados en los sitios septentrionales, Guayamba 2 (35\%) y Casa Pintada (46\%).

Esta diferencia de espesores entre las dos técnicas de talla nos posibilita inferir la idea de que el empleo de cada una de ellas se debe a la búsqueda de formas base apropiadas para la confección de determinados tipos de instrumentos. En tanto, la talla directa brinda espesores mayores ideales para producir instrumentos de ángulos más abruptos, como raspadores y raederas, la talla bipolar otorga espesores menores que brindan ángulos de bisel más agudos, propicios para producir cortantes.

\section{Reflexiones finales}

La experimentación realizada nos permitió ajustar la distinción entre los productos de distintas técnicas de talla aplicadas al cuarzo y aumentar la comprensión de los rasgos diagnósticos que presentan los productos de talla en cada caso.

Obtuvimos un mayor conocimiento sobre la materia prima, su reacción ante la talla y los productos derivados de la misma, que nos proporciona una mejor comprensión, y más detallada, a la hora de abordar nuevos estudios arqueológicos que exhiban el predominio cuarzo en la producción lítica. A futuro pretendemos seguir con las pruebas experimentales para abordar la etapa de formatización de instrumentos, y poder extender la comparación a todas las etapas productivas.

Encontramos que en el caso de la Sierra de El AltoAncasti, son el tipo de actividades realizadas y el rol que ocupaba cada asentamiento las que direccionan la producción lítica. De esta forma, no sería la posición ambiental ni la proximidad geográfica, sino el tipo de sitio lo que caracteriza la producción lítica en cada uno de los asentamientos analizados. Aunque el cuarzo presenta ciertas dificultades para la talla, los talladores de la sierra habrían encontrado una forma de trabajarlo que les permitió satisfacer sus necesidades con esta roca. La utilización de la talla bipolar pudo estar vinculada a este aspecto.

Catamarca, 20 de marzo 2019

\section{Bibliografía}

Aschero, C. (1975). Ensayo para una clasificación morfológica de artefactos líticos aplicada a estudios tipológicos comparativos. Informe al CONICET. Manuscrito inédito

Aschero, C. (1983). Ensayo para una clasificación morfológica de artefactos líticos. Apéndice $A$ y $B$. Cátedra de Ergología y Tecnología. Facultad de Filosofía y Letras. Universidad de Buenos Aires. Manuscrito inédito.

Brizuela, C. (2018). Entre gubias, escoplos y cinceles: Una caracterización funcional de base microscópica, para instrumentos líticos experimentales (Tesis de Licenciatura inédita). Universidad Nacional de Córdoba, Argentina.

De la Peña, P. (2015). A qualitative guide to recognize bipolar knapping for flint and quartz. Lithic Technology, 40(4), 316-331.

Egea, D. (2018). Tecnología lítica en la sierra de El Alto-Ancasti (Catamarca). Aporte desde la experimentación. Revista Del Museo de Antropología, 11(2), 49-58.

Gerola, I. (2018). El hábito de tallar el paisaje verde. Tecnología lítica en el sector septentrional de El AltoAncasti (Catamarca), (Tesis de Licenciatura inédita), Universidad de Buenos Aires, Argentina.

Gordillo, I., Zucarelli, V., \& Eguia, L. (2017). Las casas del sol naciente, arqueología de la vertiente oriental del Alto-Ancasti. En Ventura, B. N., Ortiz, G., \& Cremonte, M. B. (Eds.), Arqueología de la vertiente oriental surandina. Interacción macroregional, materialidades, economía y ritualidad (pp. 111-134). Buenos Aires: Sociedad Argentina de Antropología.

Knutsson, H., Knutsson, K., Taipale, N., Tallavaara, M., \& Darmark, K. (2015). How shattered flakes were used: Micro-wear analysis of quartz flake fragments. Journal of Archaeological Science: Reports,2, 517-531.

Mansur, M. E., \& Lasa, A. (2005). Diversidad artefactual vs. Especialización funcional. Análisis del IV componente de Túnel I (Tierra del Fuego, Argentina). Magallania, 33(2), 69-91.

Morgado, A., \& Baena, J. (2011). Experimentación, Arqueología experimental y experiencia del pasado en la Arqueología actual. En Morgado, A., Baena, J., García, D. (Eds.), La investigación experimental aplicada 
a la Arqueología (pp. 21-28). Granada: Universidad de Granada.

Nami, H. (2000). Investigaciones actualísticas y piedra tallada. I) Criterios experimentales para identificar lascas de talla bipolar: Su aplicación en la interpretación de artefactos arqueológicos de los extremos norte y sur de la Patagonia. En Actas del III Congreso Argentino de Americanistas 3 (pp. 229-270). Buenos Aires: Sociedad Argentina de Americanistas.

Pautassi, E., \& Sario, G. (2014). La talla de reducción: aproximaciones experimentales para el estudio del cuarzo. ArqueoWeb, 15, 3-17.

Prous, A. P., Alonso, M., Neves de Sousa, G., Pessoa Lima,
A., \& Amorel, F. (2009). La place et les caractéristiques du débitage sur enclume (« bipolaire ») dans les industries brésiliennes. Paleo, Número esp, 201-220.

Quesada, M. N., Gastaldi, M. R., \& Granizo, M. G. (2012). Construcción de periferias y producción de lo local en las cumbres de El Alto-Ancasti. Relaciones de La Sociedad Argentina de Antropología, XXXVII (2), 435-456.

Sánches Yustos, P., Diez Martín, F., Domínguez-Rodrigo, M., \& Tarriño Vinagre, A. (2012). Discriminación experimental de los rasgos técnicos en la talla bipolar y a mano alzada en lascas a través de los cuarzos de Naibor Soit (Garganta de Olduvai, Tanzania). MUNIBE (Antropologia-Arkeologia), 63, 5-26. 